\title{
Secure Transaction Processing in Firm Real-Time Database Systems
}

\author{
Binto George Jayant Haritsa \\ Supercomputer Education and Research Centre \\ Indian Institute of Science, Bangalore 560012, India \\ \{binto, haritsa\}oserc.iisc.ernet.in
}

\begin{abstract}
Mam rab-tinc datibase applications arise in safety-critical iustillations and military systems where enforcing security is r rm tal to thr surcess of the enterprise. A secure real-time dittaliase svistem has to simultaneously satisfy two requiremcul. guarantee data security and minimize the number if missicr transaction deadlines. We investigate here the (Nifurmance inplications, in terms of missed deadlines, of nuadustejug security in a real-time database system. In particular. we focus on the concurrency control aspects of fliis ismate.

( hm main comtributions are the following: First, we idenuiv which among the previously proposed real-time concurrus control protocols are capable of providing protection "hisust bul direct and indirect (covert channels) means of inamrhrrizend access to data. Second, using a detailed simulation model of a firm-deadline real-time database system, we profile the real-time performance of a representative set i) hitere secure concurency control protocols. Our expermuents show that a prioritized optimistic concurrency control protocol. OPT-WAIT, provides the best overall perforuaucc. Third, we propose and evaluate a novel dual appriacli to secure transaction concurrency control that alfin: the ral-tine datiabase system to simultaneously use diftirent concurrency control mechanisms for guaranteeing rewar and for improving real-time performance. By appropriately choosing these different mechanisms, we have brin able 10 design hybral concurrency control algorithms lhill porres avel betrer performance than OPT-WAIT.
\end{abstract}

\section{Introduction}

Many real-time database applications arise in safety-critical installaticus and military systems where enforcing security is crucial to the success of the enterprise. Surprisingly, how('ver. the issue of providing security in real-time database sisterus (RTDBS) has received comparatively little atteniwn although mid-time database research has been underwits for ckrse to a decade now. In this paper, we partially ankfress thus lacuna by making a detailed investigation of the

Permiesion to make digita/hard copy of pert or all this work for personal or cleseroom use is granted without foe provided that copies are not made or distributed for profit or commercial advantage, the copyright notice, the title of the publication and its date appear, and notice is given that copying is by permission of ACM, inc. To copy othorwise, to republish, to post on servers, or to rediatribute to liste, requires prior apecific permiesion and/or a foe. SIGMOD'97 AZ, USA

๑ 1997 ACM 0-89791-911-4/97/0005...\$3.50 performance implications of providing security in the context of real-time applications with "firm-deadlines" [9] - for such applications, completing a transaction after its deadline has expired is of no utility and may even be harmful.

\section{Database Security}

Most secure database systems have access control mechanisms based on the Bell-LaPadula model [12]. This model is specified in terms of subjects and objects. An object is a data item, whereas a subject is a process that requests access to an object. For example, when a process accesses a data file for input/output operations, the process is the subject and the data file is the object. Each object in the system has a classification level (e.g., Secret, Classified, Public, etc.) based on the security requirement. Similarly, each subject has a corresponding clearance level based on the degree to which it is trusted by the system.

The Bell-LaPadula model imposes two restrictions on all data accesses:

- A subject is allowed read access to an object only if the former's clearance is higher than or identical to the latter's classification.

- A subject is allowed write access to an object only if the former's clearance is identical to or lower than the latter's classification.

Figure 1: Bell-LaPadula access restrictions

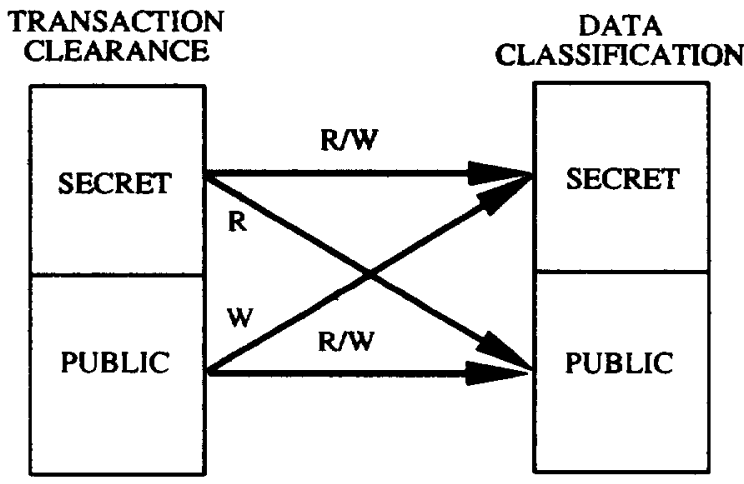

The Bell-LaPadula conditions, by enforcing a "read below, write above" constraint on transaction data accesses (an example is shown in Figure 1), prevent direct unauthorized 
acrese lo soure data. They are not sufficient, however to futer from "covert hamiels" A covert channel is an indoref mans lw which a high security clearance process can

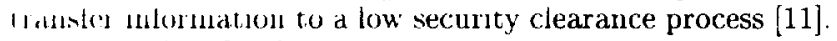
Fin rximflr. if a low serurity process requests access to "11 'xlusw' leserurce it will be delayed if the resource is ahralv hald by a high securitv process, otherwise it will imunemiately be granted the resource. The presence or absence "f rhe delay cau be used to encode information by a high security process that is conspiring to pass on information to ifw low serurity process

Ciwet diamels that use the database system's physinel rewates ar the medium for passing on information are relativh stanghtionard to tackle - for example, by intro"huciun "ucise" un the form of dummy transactions that make

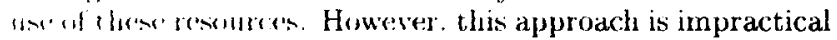
hn I wert channels that use data as the medium (for exam-

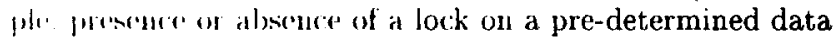
\{trui\}. This is because, unlike physical resources which are womally fen in tmuber. the ummber of data items is usuilly emommus, especially in a clatabase system. In fact, in lwavily loaded systems, noise at the physical resources may lo ginlorated "for free", but this will probably never be the rasi for data since it is trivial to insert an additional data it.un rhat is of relevance only to the conspiring transactions. 'harefore. explacitly making data access covert-channel-free 1. waw mal thath doing the same for resource access.

Covert clamuels based on data can be prevented by providimg hugher priority to the low security transaction whenenr a clata innflict occurs between a low security transacunin and a high security transaction. Taking this approach risures that lou security transactions do not "see" high serunt srasiations and are therefore unable to distinguish imern their presence or absence. This notion is formalixd in $\{6\}$ as non-interference. From a database system persurtur. It trustates to implementing a concurrency control mechanism that. supports the non-interference feature. In this paper. we quantitatively investigate the performance isuplivalisus of secure concurrency control in the context of a fim-rindline real-tine database system.

\section{Real-Time Database Security}

A sener wal-time datialiase system has to simultaneously whisly 1 wo refurements. namely, provide security and minunze the number of missed transaction deadlines. Unform11310/4. rhe mechanisms for achieving the individual goals hlon work at cross-purposes [8]. In a real-time database sincm. high prosity is usually given to transactions with ristiry drarlinus in orrler to help their timely completion. (On the other hand, in secure database systems, low security trausactions are given high priority in order to avoid covert cliannels (as described above). Now consider the situation wherin a high security process submits a transaction with a tight deadline in a secure real-time database system. In this asse. it heromes difficult to assign a priority since assigniug a high priority may cause a security violation whereas isisthing a low prority may result in a missed deadline.

Ouc approach. used by Son et al in $[4,16,17]$, to address the above problem is to adaptively tradeoff security fir timeliness depending on the state of the system. Our riw. however, is that for many applications security is an "all-or-uorthing" issue, that is, it is a correctness criterion. In conuparison, the number of missed deadlines is a performancr issue. Therefore, in our research work, we are investifat ing the problem of how to minimize the number of missed transaction deadlines without compromising security. As a first step towards achieving this goal, we have conducted a detailed simulation study to evaluate what impact the choice of concurrency control protocol has on the real-time performance. Our simulation model captures a real-time database system with an open transaction arrival process. Transactions are assigned security levels and have corresponding restrictions on their data accesses. Each transaction also has a deadline and the deadline is "firm" - that is, transactions which miss their deadlines are considered to be worthless and are "killed" (immediately discarded from the system without being executed to completion).

\section{Secure Real-Time Concurrency Control}

In recent years, several concurrency control (CC) protocols that are specially tailored for real-time database systems have been developed. These include prioritized variants of two-phase locking (2PL) such as Wait Promote and High Priority [3], and prioritized variants of optimistic concurrency control (OPT) such as Sacrifice and Wait [9]. These algorithms were primarily designed to minimize the number of missed transaction deadlines and have been evaluated on this basis in $[3,9]$.

There are significant differences between the real-time environment in which the above concurrency control algorithms were compared and the secure real-time environment. In particular, the following issues need to be considered: First, not all real-time concurrency control algorithms may satisfy the non-interference property mentioned earlier. Second, there are multiple transaction classes corresponding to the various security clearance levels. Third, the data access patterns of transactions are constrained by the BellLaPadula model. Fourth, conflicts are resolved based on both security considerations and timeliness considerations. Finally, there is the question of class fairness, that is, how evenly are the missed deadlines spread across the transactions of the various clearance levels.

Due to the above differences, the performance profiles of real-time concurrency control algorithms need to be reevaluated in the secure domain - we address this issue here.

\section{Dual Approach}

A feature of the secure environment is that there are two categories of data conflicts: inter-level and intra-level. Interlevel conflicts are data conflicts between transactions belonging to different security clearance levels whereas intra-level conflicts are data conflicts between transactions of the same level. The important point to note here is that only interlevel conflicts can result in security violations, not intralevel conflicts. This opens up the possibility of using different concurrency control strategies to resolve the different types of conflicts. In particular, we can think of constructing mechanisms such that inter-level conflicts are resolved in a secure manner while intra-level conflicts are resolved in a timely manner. The advantage of this dual approach is that the real-time database system can maximize the real-time performance, by appropriate choice of intra-level CC protocol, without sacrificing security. In contrast, the tradeoff approach mentioned earlier requires the application to compromise on security in order to achieve enhanced real-time performance. We investigate here the performance of various combinations of concurrency control mechanisms for resolving inter-level and intra-level data conflicts. 


\section{Contributions}

In this paper, we quantitatively investigate the performance insplications of guaranteeing security in a firm-deadline realInur datidadare svitem. Our main contributions are the follimilig

1 Wi identify which among the previously proposed real-time concurrency control protocols are capable of providing protection against both direct and indiwel (corert diannels) means of unauthorized access to data. That is, which protocols support the concept of nors-interference.

2. Csing a detailed simulation model of a firm-deadline real-time database system, we profile the real-time perlurmance of a representative set of secure concurrency control protocols. Our simulations consider a variety of security-classified transaction workloads and system ronfigurations. To isolate and quantify the performance effects of supporting covert channel security, we also evaluate the performance of the $\mathrm{CC}$ protocols in the context of a baseline system that prevents direct ullathorized arcess, but not covert channels (that is, it only supports the Bell-LaPadula restrictions). Our experiments show that a prioritized optimistic concurrency control protocol, OPT-WAIT, provides the best werall performance.

3. Wo cvaluate the effectiveness of a novel dual approach to secure transaction concurrency control wherein siimitianermsly different CC mechanisms are used for guaranteeing security and for improving real-time performance. respectively. In particular, we investigate the performance of various combinations of concurrency control mechanisms for resolving inter-level and intra-level data conflicts. Our results show that some if these hybrid concurrency control algorithms perform

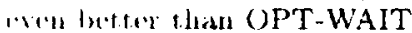

\section{Related Work}

The design of secure CC protocols in the context of conm'ntumul clatabase systems has been investigated by sevral research groups (see [20] for a survey). In comparisom. little attention has been given to developing secure $C C$ potocols for real-time database systems. The only work that we are aware of in this area is a series of papers by Sul "t al $[4,13.16,17,19]$. In particular, a concurrency routrol protocol that attempts to balance the dual requiresus'sit of servirity and timeliness is presented in $[4,16,17]$. In thry schenc. transartions dynamically choose between $2 \mid \cdot L-H P\{3\}$ an (unsecure real-time version of $2 P L$, and $\left.S 2 l^{\prime}\right\}$. [15]. a secure (non-real-time) version of $2 \mathrm{PL}$. The goal

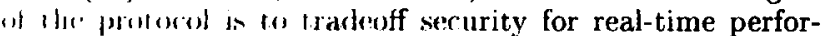
ulass with the tradeoff depending on the state of the systein and the application's requirements. ${ }^{1}$ In contrast, in our work. we have assumed that full security is a fundamental mpinmureut and that it is not permissible to improve the mil-11me performance at the cost of security.

III [13], a concurrency control protocol that ensures both wourty and timcliness is proposed. For this scheme, howver. the RTDBS is required to maintain two copies of each

'Fire irackolf approach, and alternative schemes to implement the

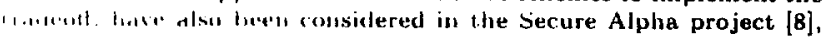

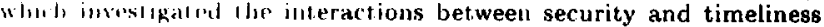
II the collle'xi ol at chistributed real-time operating system. data item. Further, transactions are required to obtain all their data locks before starting execution (i. e., strict static locking). These requirements limit the applicability of the protocol. In our work, we consider more general database environments where all data items are single-copy and transactions acquire data locks dynamically.

Another feature of their work is that it is primarily addressed towards "soft-deadline" applications, that is, realtime applications in which there is value to completing tasks even after their deadlines have expired. In contrast, we have concentrated on firm-deadline applications. The type of deadline has a significant impact on both the performance evaluation model and on the interpretation of the results, as observed earlier for (unsecure) real-time transaction concurrency control $[3,9]$.

\section{Secure Concurrency Control Protocols}

As mentioned in the Introduction, assigning priorities in a secure real-time database system is rendered difficult due to having to satisfy multiple functionality requirements. In our study, since we assume that security is a correctness requirement, the database system is forced to assign transaction priorities based primarily on security clearance levels and only secondarily on deadlines. In particular, we assign priorities as a vector $P=$ (LEVEL, INTRA), where LEVEL is the transaction security clearance level and INTRA is the value assigned by the priority mechanism used within the level. We assume that security levels are numbered from zero upwards, with zero corresponding to the lowest security level. Further, priority comparisons are made in lexicographic order with lower priority values implying higher priority.

With the above scheme, transactions at a lower security level have higher priority than all transactions at a higher security level, a necessary condition for non-interference. For the intra-level priority mechanism, any priority assignment that results in good real-time performance can be used. For example, the classical Earliest Deadline assignment where transactions with earlier deadlines have higher priority than transactions with later deadlines. In this case, the priority vector would be $P=$ (LEVEL, DEADLINE).

In conjunction with the above priority assignment, it would seem at first glance that, in principle, any real-time concurrency control protocol could be used in a secure RTDBS and that the actual choice of protocol would be based only on the relative performance of these protocols. However, not all the previously proposed real-time $C C$ algorithms are amenable to supporting security requirements. For example, consider the 2PL Wait Promote algorithm proposed in [3]: This protocol, which is based on 2PL, incorporates a priarity inheritance mechanism [18] wherein, whenever a requester blocks behind a lower-priority lock holder, the lock holder's priority is promoted to that of the requester. In other words, the lock holder inherits the priority of the lock requester. The basic idea here is to reduce the blocking time of high priority transactions by increasing the priority of conflicting low priority lock holders (these low priority transactions now execute faster and therefore release their locks earlier).

The Wait Promote approach is not suitable for secure real-time database systems. This is because it permits the blocking of high priority transactions by low priority transactions which violates the requirement of non-interference between the transactions of different security levels (as mentioned in the Introduction, non-interference means that low 
somus tantsactions should not be able to distinguish bemoen the presence or absence of high security transactions).

To generalize the above observation, a real-time CC protorol that pcrmits, to even a limited extent, high priority transactions to be adversely affected by low priority transil tiums. a plenomenon known as priority inversion in the ral-1unc litcrature [18], cannot be used in a secure RTDBS. Iprat frum llius Prumote, other examples of real-time CC niguthlum than fall into this category include 2PL-CR [3], 2]'L-( )S/BI [2] aud WAIT-50 [9].

lis the rmanular of this section, we briefly present a

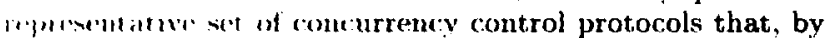

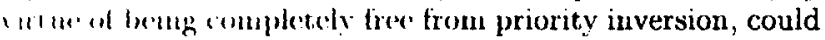
he "tsid to resolve conflicts in a secure real-time database istoull Thuse protocols use either locking or optimistic nurureney coutrol as the basic regulatory mechanism.

\subsection{PL High Priority}

The 2PL High Priority (2PL-HP) scheme [3] modifies the "bassical striet two-phase locking protocol (2PL) [5] by incor-

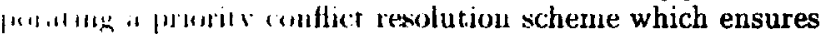
llas bugh prusitu trausartions are not delayed by low priurit transactions. In 2PL-HP, when a transaction requests a hork on a datia item that is held by one or more higher munily mansactions in a conflicting lock mode, the requesting transaction waits for the item to be released (the wait "pun kus at slatia item is managed in priority order). On the whos haud, if the data item is held by only lower priority mansactions in a conflicting lock mode, the lower priority housartions are restarted and the requesting transaction is granted the desired lock. ${ }^{2}$ Note that 2PL-HP is inherently dracliock-free if priorities are assigned uniquely (as is usually the kate in real-time database systems).

\subsection{OPT-SACRIFICE}

Thr (IPT-SACRIFICE algorithm (9) modifies the classical

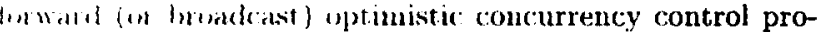

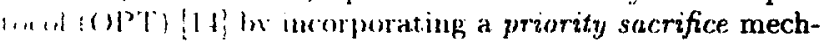
musm In this algorithm, a transaction that reaches its viliclinsun stings diresks for conflicts with currently executing lanisarisoms. If conthicts are detected and one or more l'ansialsous in the conflict set is a higher priority transacIIIII. the' the validating transaction is restarted - that is, It is writiced in an effort to help the higher priority transartions make their deadlines. Otherwise, the transaction is illuwed tw commit. restarting in the process the lower pri-

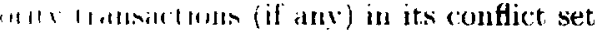

\subsection{OPT-WAIT}

The oPT.WIT algurithu [9] modifies the forward OPT protocol by incorporating a priority wait mechanism. Here, " lausastion that reaches validation and finds higher prioriI 1 transactions in its conflict set is "put on the shelf", that is. it is makl to wait and not allowed to commit immediacly. This gives the higher priority transactions a chance wis liake their deadlines first. While a transaction is waiting un the shelf, it is possible that it may be restarted due to the commit of one of the conflicting higher priority transartions. If at ally time during its shelf period, the waiting "Iussurtun fiucls uo higher priority transactions remaining III its couflict set, it is committed, restarting in the process lhe lower priority transactions (if any) in its conflict set.

\footnotetext{
1 ine" reituler in allowed th joill at group of lock-holding readers
}

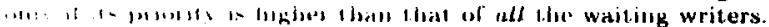

\section{$3.4 \quad \mathrm{~S} 2 \mathrm{PL}$}

A secure locking-based protocol called Secure 2PL (S2PL) was recently proposed in [15]. The basic principle behind Secure $2 \mathrm{PL}$ is to try to simulate the execution of conventional 2PL without blocking the actions of low security transactions by high security clearance transactions. This is accomplished by providing a new lock type called virtual lock, which is used by low security transactions that develop conflicts with high security transactions. The actions corresponding to setting of virtual locks are implemented on private versions of the data item (similar to optimistic concurrency control). When the conflicting high security iransaction commits and releases the data item, the virtual lock of the low security transaction is upgraded to a real lock and the operation is performed on the original data item. To complete this scheme, an additional lock type called dependent virtual lock is required apart from maintaining, for each executing transaction $T_{i}$, lists of the active transactions that precede or follow $T_{i}$ in the serialization order. The complete details are given in [15]. ${ }^{3}$

Note that Secure 2PL may not perform well in the realtime domain since it does not include any real-time-specific features. We include it here for two reasons: First, it serves as a baseline against which to compare the real-time CC algorithms. Second, we use it in one of the "dual approach" protocols evaluated in this study.

\section{Dual Approach}

In this section, we move on to discussing our new dual approach to secure real-time concurrency control. As mentioned in the Introduction, a feature of the secure environment is that there are two categories of conflicts: inter-level and intra-level. This opens up the possibility of using different concurrency control strategies to resolve the different types of conflicts. In particular, we can think of constructing mechanisms such that inter-level conflicts are resolved in a secure manner while intra-level conflicts are resolved in a timely manner. For example, S2PL could be used for interlevel conflicts while OPT-WAIT could be used to resolve intra-level conflicts. The advantage of this dual approach is that the real-time database system can maximize the realtime performance without sacrificing security.

At first glance, it may appear that using multiple concurrency control mechanisms in parallel could result in violation of the transaction serializability requirement. This could happen, for example, if the serial orders enforced by the individual mechanisms were to be different. A detailed study of a generalized version of this problem is presented in [21], wherein the transaction workload consists of a mix of transaction classes and the objective is to allow each transaction class to utilize its preferred concurrency control mechanism. They propose a database system architecture wherein intraclass conflicts are handled by the class's preferred concurrency control manager while inter-class conflicts are handled by a new software module called the Master Concurrency Controller (MCC) that interfaces between the transaction manager and the multiple concurrency control managers. The MCC itself implements a complete concurrency control mechanism. A single global serialization order is ensured in the entire database system by using a Global Ordering

\footnotetext{
${ }^{3}$ In our implementation, we have had to partially modify Secure 2PL since the algorithm (as described in [15]) does not eliminate noninterference under all circumstances - the details of the modifications are available in [7].
} 
Table 1 simulatum Model Parameters

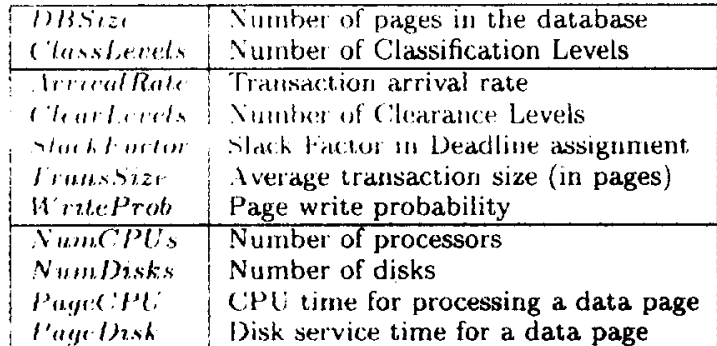

Sheme. The drtails of this scheme and the proof of its correctmess are given in [21].

For our study, we assume use of the above architecture. In this trmumork. a S2PL/OPT-WAIT combination, for eximulc. would correspond to using S2PL at the Master Conrurrancy (ontroller for resolving inter-level conflicts, and using (PPT-WAIT as the local concurrency controller within "'wh serurity lesel for resolving intra-level conflicts.

\section{Simulation Model}

lu the merkus section, we discussed various secure concurrma commol protocols. To evaluate the real-time performinus' of these algorithms, we developed a detailed simufirinu umsicl of a firm-deadline real-time database system, similar to that described in [9]. A summary of the key model parameters is given in Table 1

In ent nurled the sytem consists of a shared-memory unulumusisurir DUMS operating on disk-resident data (for simplicity. we assume that all data is accessed from disk ind buffer pool cousiderations are therefore ignored). The databuse is modeled as a collection of DBSize pages that arr uniformly randomly distributed across all of the disks. Thre ratrabisise is equally partitioned into ClassLevels securiv Masification levels (for example, if the database has I IMII yages and the number of classifications is 5, pages 1 through 200 belong to level 1 , pages 201 through 400 belong 10 level 2, and so on). Transactions are generated in a l'bisem strean with rate ArrivalRate and each transaction Lits an insociated security clearance level and a firm comfilction deadline. A transaction is equally likely to belong (1) ans if th' Cleorlevels security clearance levels. (For -mulicits. Wo issum in this study that the categories (e.g., Sirsol. Pulblic) for datid classification and transaction clear-

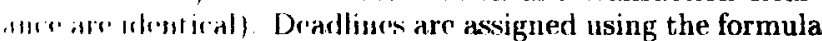
$U_{i}=H_{1}+S F * R_{T}$. wherr $D_{T}, A_{T}$ and $R_{T}$ are the deadline, arrival tume and resource time, respectively, of transaction $T$. while $S F$ is a slack factor. The resource time is the total sirwe line at the resources that the transaction requires fir) its data processing. The SlackFactor parameter is a rimstiult that provides control over the tightness/slackness of transaction deadines.

A transaction consists of a sequence of page read and frige write accesses. The number of pages accessed by a inusulun vartes uniformly between half and one-and-ahiall tum's the value of TransSize. The WriteProb paramerter cletermines the probability that a transaction opr'snson is a write. Due to security reasons, each transac11m call only acresis data from a specific segment of the tatiabasc, and page requests are generated by uniformly ranchuly sampling (without replacement) from the database over this range. The permitted access range is determined by both the security clearance level of the transaction and the desired operation (read or write), and is according to the Bell-LaPadula specifications: a transaction cannot read (resp. write) pages that are classified higher (resp. lower) than its own clearance level. A transaction that is restarted due to a data conflict has the same clearance level, and makes the same data accesses, as its original incarnation. If a transaction has not completed by its deadline, it is immediately killed (aborted and discarded from the system).

A transaction read access involves a concurrency control request to get access permission, followed by a disk $1 / O$ to read the page, followed by a period of CPU usage for processing the page. Write requests are handled similarly except for their disk $1 / O$ - their disk activity is deferred until the transaction has committed. We assume that the RTDBS has sufficient buffer space to allow the retention of updates until commit time.

The physical resources of the database system consist of NumCPU's processors and NumDisks disks. There is a single common queue for the CPUs and the service discipline is Pre-emptive Resume, with preemptions being based on transaction priorities. Each of the disks has its own queue and is scheduled according to a Head-Of-Line (HOL) policy, with the request queue being ordered by transaction priority. ${ }^{4}$ The PageCPU and PageDisk parameters capture the CPU and disk processing times per data page, respectively.

\section{Experiments and Results}

In this section, we present the performance results from our simulation experiments comparing the various secure CC protocols in a firm-deadline RTDBS environment. The primary performance metric of our experiments is MissPercent, which is the percentage of input transactions that the system is unable to complete before their deadlines. We compute this percentage on a per-clearance-level basis also. MissPercent values in the range of 0 to 20 percent are taken to represent system performance under "normal" loads, while MissPercent values in the range of 20 percent to 100 percent represent system performance under "heavy" loads [9]. Only statistically significant differences are discussed here [7].

An additional performance metric is ClassFairness which captures how evenly the missed deadlines are spread across the transactions of the various clearance levels. To compute this we use, for each class $i$, the formula Fairness $s_{i}=$ CommitTrans CommitTrans/InputTrans $_{i}$. In this formula, CommitTrans and InputTrans $i$ are the number of committed transactions and the number of input transactions, respectively, of class $i$, while CommitTrans and InputTrans are the total number of committed transactions and the total number of input transactions, respectively, across all classes. With this formulation, a protocol is ideally fair, if the fairness value is 1.0 for all classes. Fairness values greater than one and lesser than one indicate positive bias and negative bias, respectively.

The transaction priority assignment used for the secure protocols in the experiments described here is $P=$ (LEVEL, DEADLINE), thereby ensuring that there are no covert channels since a low security transaction is delayed only by transactions of its own level or those of lower security levels.

${ }^{4}$ For simplicity, our model uses the non-interference method for eliminating covert channels at the physical resources also - an alternative method is the "noise" technique mentioned in the Introduction. 
Cahlo 2. Baselint Parameter Settings

\begin{tabular}{|c|c|c|c|}
\hline 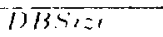 & 1060) pages & NumCpus & 10 \\
\hline $11, \ldots 1.1,1$. & 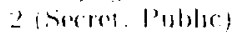 & Numbisks & 20 \\
\hline 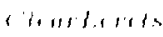 & $\because 1$ arout frublac & Payec:PU & $10 \mathrm{~ms}$ \\
\hline vinatherelos & 1.0 & PrigeDisk: & $20 \mathrm{~ms}$ \\
\hline $1 \cdot 11,4,: 1$ & 11, 1,121:s & & - \\
\hline $11, \ldots 1,11, d$, & 11.7 & & \\
\hline
\end{tabular}

\subsection{Comparative Protocol}

Tis hilp isolate and understand the performance cost that incurs due to having to eliminate covert channels, we have itse simmlated the performance achievable in the absence of curme rasmus serusity requirements. That is, the perforIlatue alluevable if only Bell-LaPadula conditions had to he sur isfichl. For this scenario, a priority assignment of $P=$ DEADLINE is unerl. In the following experiments, we will rofer to the performance achievable under this scenario as DIRECT sinc the Boll LaPadula conditions prevent diren man homed acess to data.

\subsection{Experiment 1: Resource and Data Contention}

The sittings of the workload parameters and system paramcters for our first experiment are listed in Table 2. These sttmings were chosen with the objective of having significant (litia contemtion and resource contention in the system, thus firlping $k_{3}$ brung ont the performance differences between the ravins roncurrency control protocols.

in thm experiment, there are two security levels: Secret raul Puhlue Fon this svitem. Figures $2 \mathrm{a}$ and $2 \mathrm{~b}$ show the

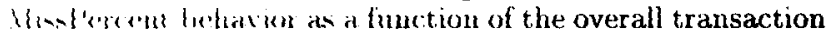

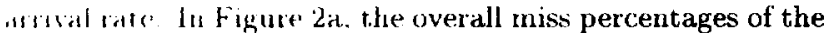
lull sertre algurithms and their DIRECT (Bell-LaPadula) winumipartis is protiled. Wo see here that at normal loads thr performanse of the secure algorithms is worse than that 1) their DIRECT counterparts. In contrast, under heavy loaks the performance of the secure algorithms is actually better than that of the DIRECT algorithms. The reason for this is that while the Earliest Deadline priority assignnu'nt is cxcrllent for a set of tasks that can be completed

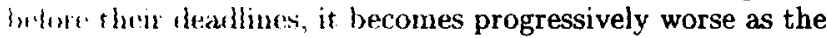
lask set overloads its capacity [10]. In this situation, the sceme protercols feature of grouping the transactions into miuritized levels means that Earliest Deadline is operational "uliw smaller set of transactious, leading to improved perfusmance al higher loads. In summary, although elimination if conrel chamels results in performance degradation at normal lorris, is rerluces the miss percentage under heavy loads.

bucusing on the secure real-time algorithms, we observe fiis) III Figure 2a that the performance of 2PL-HP is signif"andly worse than that of the optimistic algorithms, OPTWAIT and OPT-SACRIFICE (denoted by OPT-SCR in the l'gr'md). In fact, 2PL-HP's performance is no better than that of S2PL which. as mentioned in Section 3, is a nonrat-time protecol! The poor performance of 2PL-HP is primmily berause of it.s "wasted restarts" problem, which "1, 11 main drawbark in unsecure real-time CC also [9]: 1 1ransulum mav he restarted bv a higher priority trans-

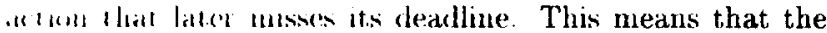
restas dicl sut scrubl in the higher priority transaction meetMu 11 - dikllun. In addition. it may cause the lower priority transatimu to miss its deadline as well, apart from wasting

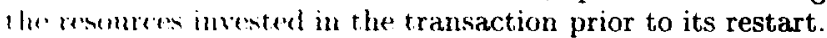

The effect of the wasted restarts problem is magnified in the secure domain for the following reason: In unsecure realtime CC, a transaction that is close to its deadline would usually not be restarted since it would have high priority. However, in the secure model, where the transaction level is also a factor in the priority assignment, Secret transactions that are close to their deadlines may still be restarted due to data conflict with a Public transaction.

Moving on to OPT-SACRIFICE, we find that there is a change of performance behavior in the secure environment in that the gap between its performance and that of 2PL-HP is more than that observed for unsecure real-time $\mathrm{CC}$ [9]. The main problem for OPT-SACRIFICE in the unsecure domain was that it suffered from "wasted sacrifices" (sacrifices for a transaction that is eventually killed). The effect of this problem is diminished in the secure domain due to the access pattern restrictions of the Bell-LaPadula model: The definition of conflict in forward optimistic concurrency control is that a conflict exists between the validating transaction $V$ and an executing transaction $E$ if and only if the intersection of the write set of $V$ and the current read set of $E$ is non-empty. For the LaPadula model, where blind urites are permitted due to the "read-below, write-above" paradigm, optimistic algorithms will correctly conclude that there is no conflict between items that are in the intersection of the write set of $V$ and the write set of $E$ but not in the read set of $E$. In fact, it is easy to see that a validating Secret transaction will never have conflicts with executing Public transactions in this model. Therefore, the possibility of wasted sacrifices decreases as compared to the unsecure domain. Note that for 2PL-HP, however, blind-writes can unnecessarily result in write-write conflicts and cause either blocking or restarts, thereby further deteriorating its performance.

Turning our attention to OPT-WAIT, we see that it provides the best performance across the entire loading range. This is because it derives, similar to OPT-SACRIFICE, the above-mentioned benefits arising out of the Bell-LaPadula access restrictions. In addition, it suffers neither from wasted restarts nor from wasted sacrifices. Instead, all restarts are useful in that they are made "on demand" and at the commit time of a higher priority transaction.

Finally, moving on to S2PL, we find that it manages to perform on par with 2PL-HP in spite of not being deadline cognizant. This is due to its "optimistic-like" feature of virtual commit, which considerably reduces the amount of blocking associated with 2PL. This phenomenon is similar to that seen in [9], wherein a conventional (non-real-time) optimistic protocol performed better than locking-based realtime protocols.

In Figure 2b, we present the miss percentages of the various concurrency control protocols on a per-security-level basis. This graph clearly shows how the high-security Secret transaction class (dashed lines) suffers much more than the Public transaction class (dotted lines) to satisfy the goal of avoiding covert channels. Figure 2c provides statistics about the corresponding breakup of the "restarts ratio" (the average number of restarts of a transaction) on a level basis. We see here that Secret transactions are restarted much more often than Public transactions under normal loads. Under heavy loads the number of restarts decrease for Secret transactions since resource contention, rather than data contention, becomes the more dominant reason for these transactions missing their deadlines.

In Figure 2d, we present a different view of the transaction restarts picture. Here, the restarts of Secret trans- 
Figure 2a: Covert security cost

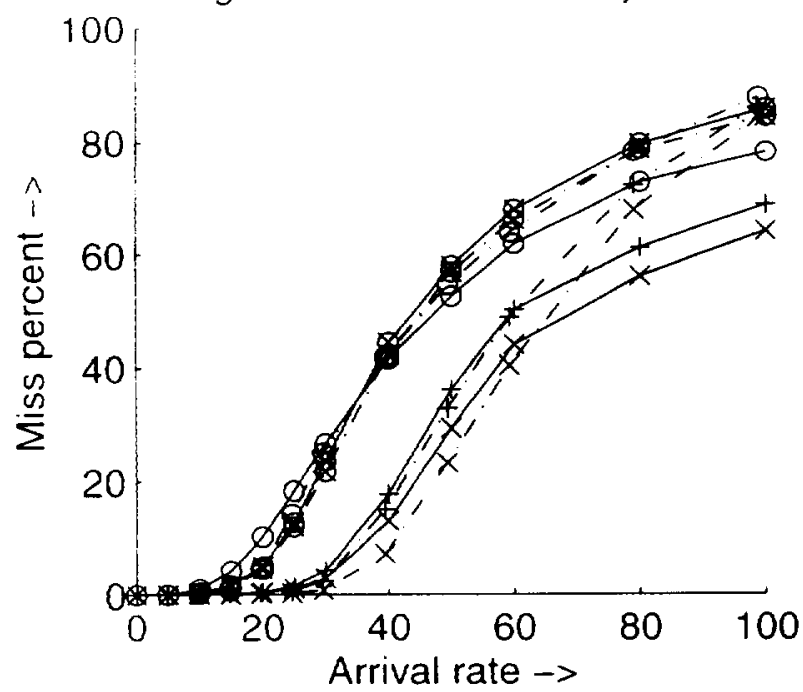

Figure 2c: Level restart ratio

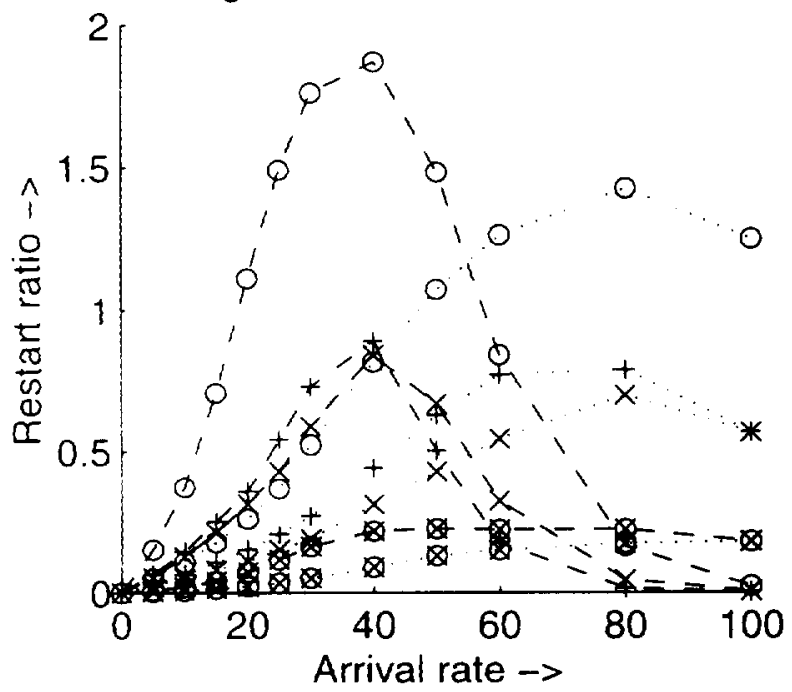

Figure 2e: Fairness

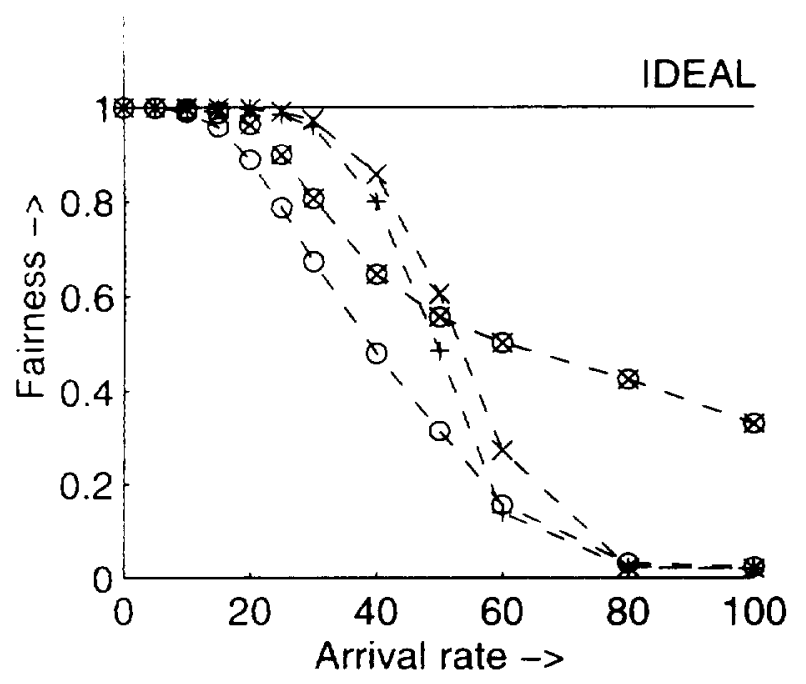

Figure 2b: Level miss percent

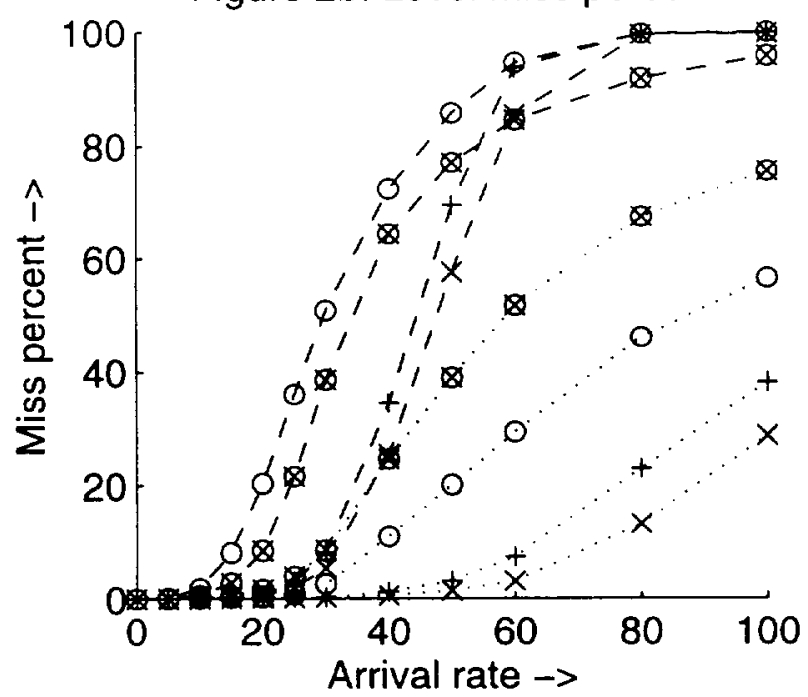

Figure 2d: Secret restart ratio

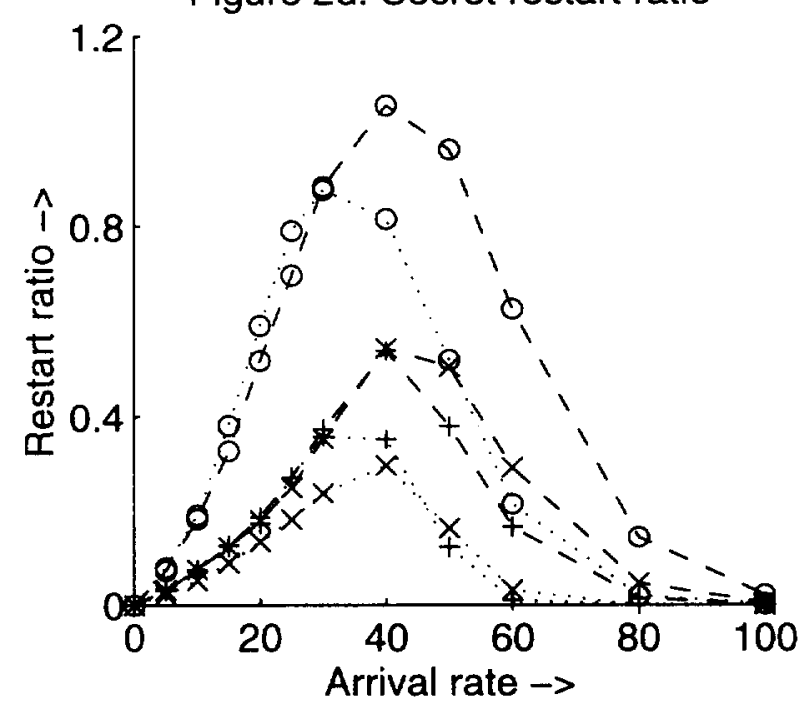


Figure 3a: Covert security cost

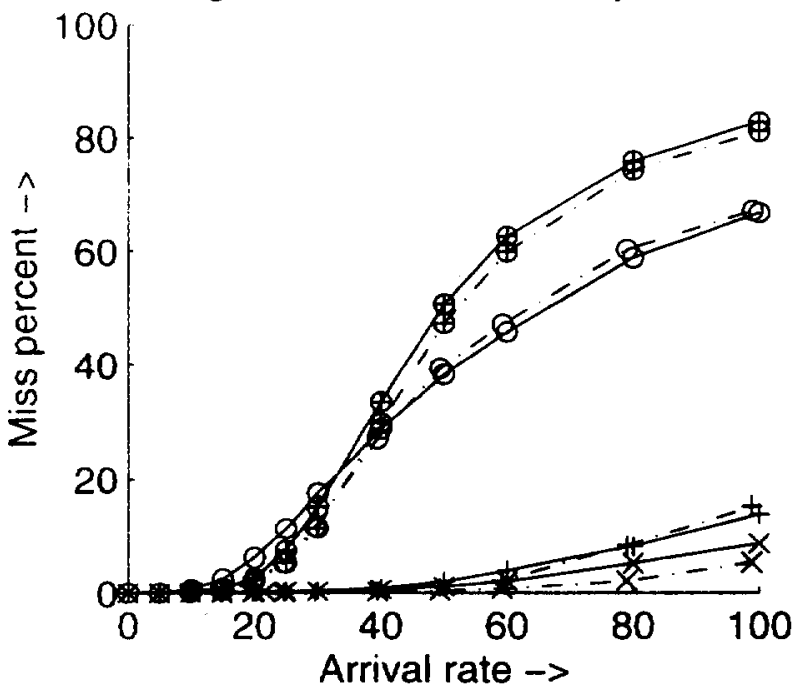

Figure 3c: Fairness

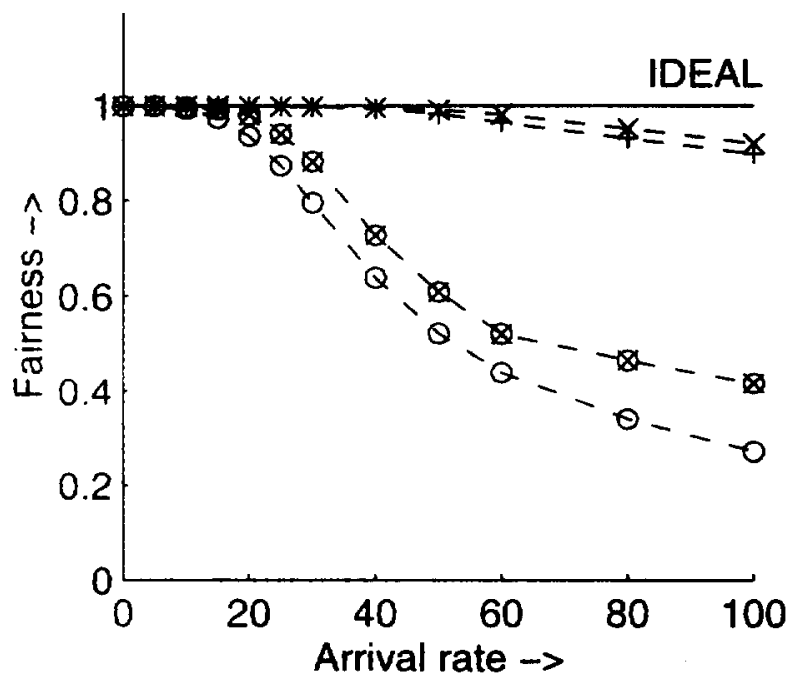

actious are (atesgorized into those caused by Public transartiour (i. (.. inter-leqel restarts) and those caused by Se(wet trusistions (i. e., intra-level restarts). Note that this lwrikup is uscauiugful ouly for Secret transactions since all mstati are intra-level for Public transactions. The graph 1. harly slows that Secret transactions suffer more from interlevel conflicts (dashed lines) than from intra-level conflicts (dot, led lines) over most of the loading range.

Finally, in Figure 2e, we plot the faimess factor of each C $C$ protocol for the Secret transaction class. We observe that at light. loads when virtually all transactions make their riadlines. all the concurrency control protocols are (trivtallv) fair. As the loading increases, however, the proto"wh- become increasingly unfair since they selectively miss llur iradlines of Secref transactions to accommodate the luhlu ransactions. With regard to the relative fairness

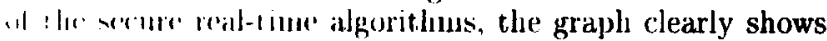
Itai (I) T-WAIT and OPT-SACRIFICE provide much betIw farluess than 2PL-HP, and that OPT-WAIT is the best wivill fi. nall secm in Figure 2e that, at high loads, S2PL is more fair than OPT-WAIT. Note, however, that this is
Figure 3b: Level miss percent

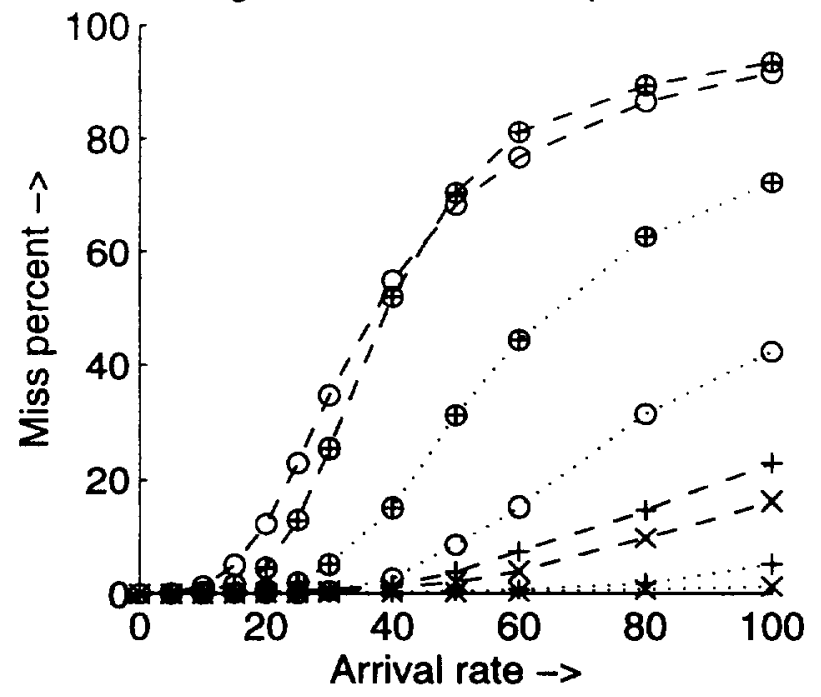

Fig. 3a

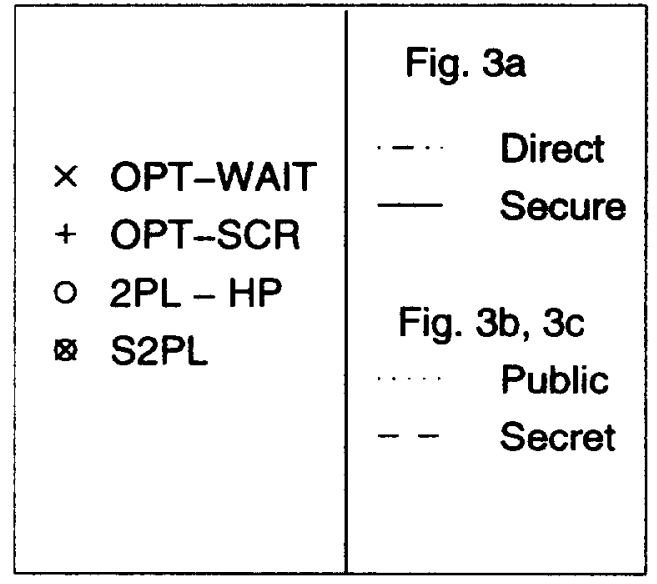

really a "virtual fairness" since it arises out of S2PL, due to its non-real-time nature, missing a large fraction of the Public transactions, rather than out of meeting the deadlines of more Secret transactions.

In summary, for the workload and system configuration considered in this experiment, OPT-WAIT provides the lowest miss percentage, both on an overall basis and on a perlevel basis, and the best overall fairness.

\subsection{Experiment 2: Pure Data Contention}

The goal of our next experiment was to isolate the influence of data contention on the performance of the concurrency control protocols. For this experiment, therefore, the resources were made "infinite", that is, there is no queueing for these resources [1]. The remaining parameter values are the same as those used in the baseline experiment. The performance results for this system configuration are presented in Figures 3a through 3c. We observe in these figures that the differences in the relative performance of the various protocols increases as compared to those seen in the previous 
'xprument. The overall (Figure 3a) as well as the per-level (Finde 3h) miss percentages of OPT-WAIT are consideraible betre than those of 2PL-HP. Here, OPT-WAIT does Inlla thall 2PL-HP for two reasions: First, the basic wasted resiats mollen of 2PL-HP occurs here too and is magnilied the 10 lla higher level of data contention. Second, the blukmg cumponcut of 2PL-HP reduces the number of trans111mus that are naking progress in their execution. This blukisn cans's an increase in the average number of transw. Toms in the system, thus generating more conflicts and a nreater number of restarts. With OPT-WAIT, however, n ransactions are never blocked for data access.

Hining on to the performance of OPT-SACRIFICE, we chserve that its performance becomes even closer to that of OPT-WAIT as compared to the previous experiment. The "risisn for this in that the wasted utilization arising out of its wased sacritices problem has no impact here since resource inllentinll is lunt ant issille.

Furll, m Figur. 3c. Which profiles the fairness factors (1) the protocols: (3PT-WAIT and OPT-SACRIFICE are alumol inlowlle fair wer most of the loading range since they mis, wr tew cleadlines overall. In contrast, 2PL-HP and

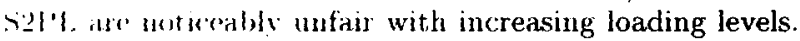

\subsection{Experiment 3: Increased Security Levels}

A two-security-level system was modeled in the previous expreriments. In our next experiment, we investigated the 'M'formanc: behavior for a five-security-level system, where the lavels are TopSecret, Secret, Confidential, Classified and Puhlir The remaining parameter values are the same as thowe used in Experiment 1. The results for this experiment am" shown in Figures 4a through 4e (for graph clarity, the rsults fur unly 2PL-HP and OPT-WAIT are presented).

In Figure 4d, we see that there is a greater difference lur wr'n the performance of the secure algorithms and their DIILECT counterparts at normal loads, as compared to the içuralent two-level experiment (Experiment 1). This is be" ritis' $n$ a tive-level system, priority is much more level-based than cleadline-baned. thereby denying Earliest Deadline its Hinlity to complete inost transactions in a feasible set under uorural loads. Under heavy loads, however, the smaller sizes of tlie transaction sets in each level results in Earliest Deadlim pertorming well for the low security transactions. (This liattur of Earliest Deadline was used in the Adaptive Earlu's [Mralliur schoduling algorithm described in [10] where Iransations are split up into prioritized groups with the size "if the highess priority group set equal to an estimate of the maxumun sumber of ransastions that could be successfully (muphered br Earliest Deadline.)

II Figures thand 4c, we plot the miss percentage on a mer-serurity-level basis for OPT-WAIT and 2PL-HP, respecIIwis. Hoces graphs dearly show the extent to which the mm prentages are skewed among the various transaction we'urity levels, with Top Secret transactions having the most muuber of uissed deadlines and Public transactions having the least. The graphs also show that OPT-WAIT's performance is hetter than that of 2PL-HP for every transaction sermurity level.

In Figures $4 d$ and $4 e$, the fairness factors for the top four sururty levels (Top Secret. Secret, Confidential and Classificd) are plotted on a per-security-level basis for OPT-WAIT aud 2PL-HP. respectively. These figures clearly show that in the luading factor increases, progressively more and more sururitv classes become discriminated against by the lowest s' unu rlasis (Public). We also find that OPT-WAIT's per- formance is more fair than that of 2PL-HP for every transaction security level.

In summary, just as in the two-security-level experiment, we find that OPT-WAIT provides the lowest miss percentage, both on an overall basis and on a per-level basis, and the maximum fairness (this observation regarding OPT-WAIT is true also with regard to the OPT-SACRIFICE and S2PL protocols whose results were not presented here).

\subsection{Experiment 4: Dual Approach}

As mentioned earlier, we have experimented with a dual approach to secure real-time concurrency control where interlevel conflicts are handled by one protocol while intra-level conflicts are handled by a different protocol. In Figures $5 \mathrm{a}$ through $5 c$ we show the performance of three dual systems for the same environment as that of Experiment 1. The combinations (in inter/intra order) are 2PL-HP/OPT-WAIT, OPT-WAIT/2PL-HP, and S2PL/OPT-WAIT, which we will refer to as HP-WAIT, WAIT-HP, and S2PL-WAIT, respectively. For the sake of comparison, the performance of a pure OPT-WAIT protocol is also shown in these graphs.

Focusing our attention on the WAIT-HP and HP-WAIT dual protocols, we first observe in Figure $5 \mathrm{a}$, which compares the overall miss percentages of the protocols, that the performance of both these approaches is considerably worse than that of the pure OPT-WAIT protocol. The reason that OPT-WAIT remains the best among them is that 2PL-HP is a wasteful algorithm, as seen in the previous experiments, and therefore "dilutes" the effect of OPT-WAIT in both the dual protocols.

We also observe in Figure 5a that that the performance of WAIT-HP is worse than that of HP-WAIT throughout the loading range. The reason for this is that, in the secure system, the number of intra-level conflicts are significantly more than the number of inter-level conflicts. Therefore, the algorithm which is used to handle intra-class conflicts has more effect on the overall miss percentage than the protocol used to handle inter-class conflicts. In WAIT-HP, it is 2PLHP which handles intra-class conflicts and this results in worse performance than that of HP-WAIT, which uses OPTWAIT to handle this category of conflicts.

The miss percentages of the protocols on a per-securitylevel basis is provided in Figure $5 \mathrm{~b}$ and the fairness factors are shown in Figure 5c. An interesting feature in these graphs is that at high loads, the fairness of WAIT-HP is greater than that of OPT-WAIT - this is the first time in all the experiments discussed so far that a real-time protocol has improved on OPT-WAIT's fairness performance. The reason that this happens is the following: In WAIT-HP, due to 2PL-HP being used for intra-class conflicts, many of the Public transactions are so busy "fighting" each other that they don't ever reach the end of their execution, which is when the OPT-WAIT policy of checking for inter-class conflicts comes into play. Therefore, Secret transactions suffer much less restarts from the Public transactions. This is clearly seen in Figure $5 \mathrm{~b}$ where the miss percentage of the Public transactions for WAIT-HP is quite high as compared to the corresponding numbers for the other protocols.

Moving on to the S2PL-WAIT dual protocol, we find that, unlike the other two dual protocols, it performs better than OPT-WAIT, especially at lower loading levels. For example, at an arrival rate of $\mathbf{4 0}$ transactions per second, S2PL-WAIT more than halves the miss percentage suffered by OPT-WAIT (Figure 5a). The reason that this combination works well is that Secure 2PL handles inter-class con- 
Figure 4a: Covert Security Cost

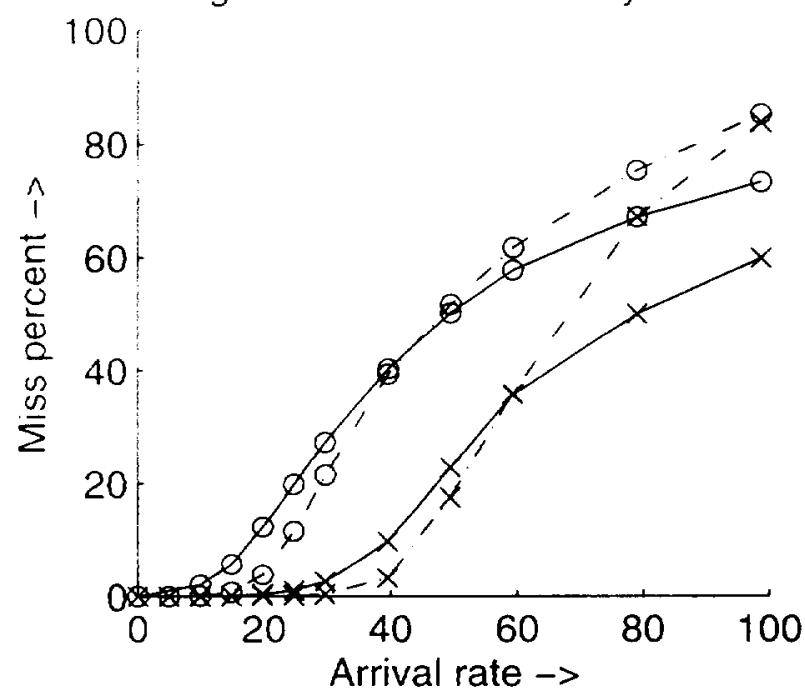

Figure 4c: Level wise (2PL-HP)

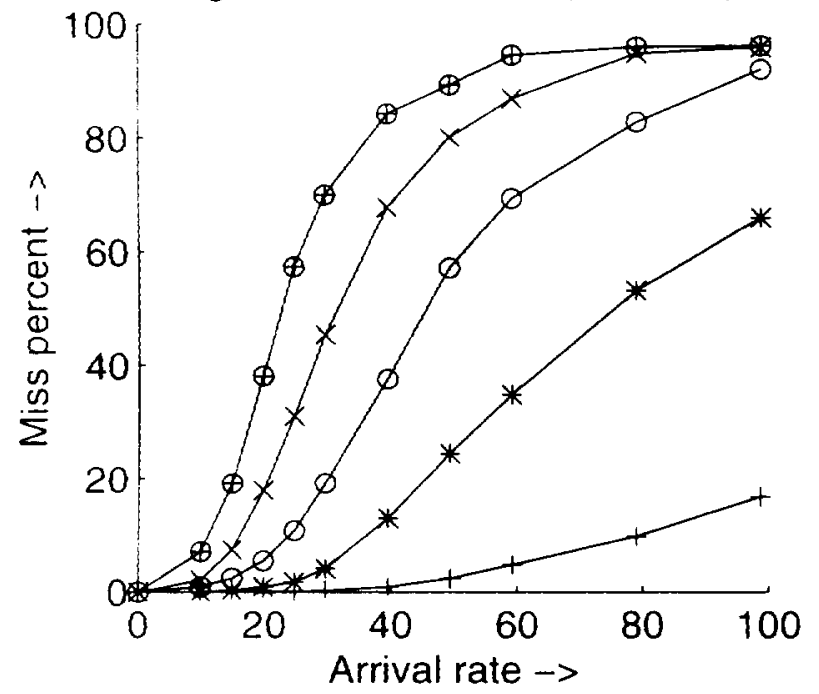

Figure 4e: Fairness (2PL-HP)

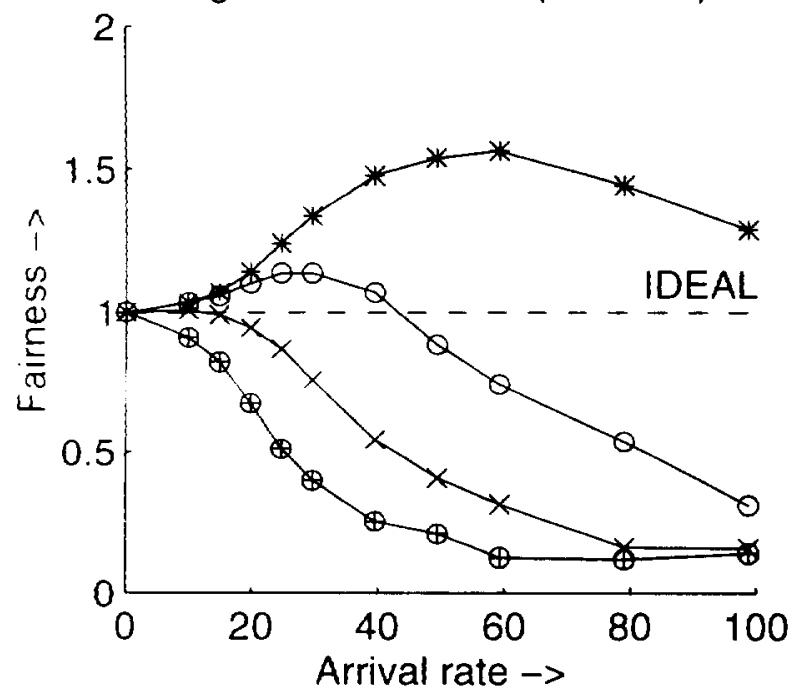

Figure 4b: Level wise (OPT-WAIT)

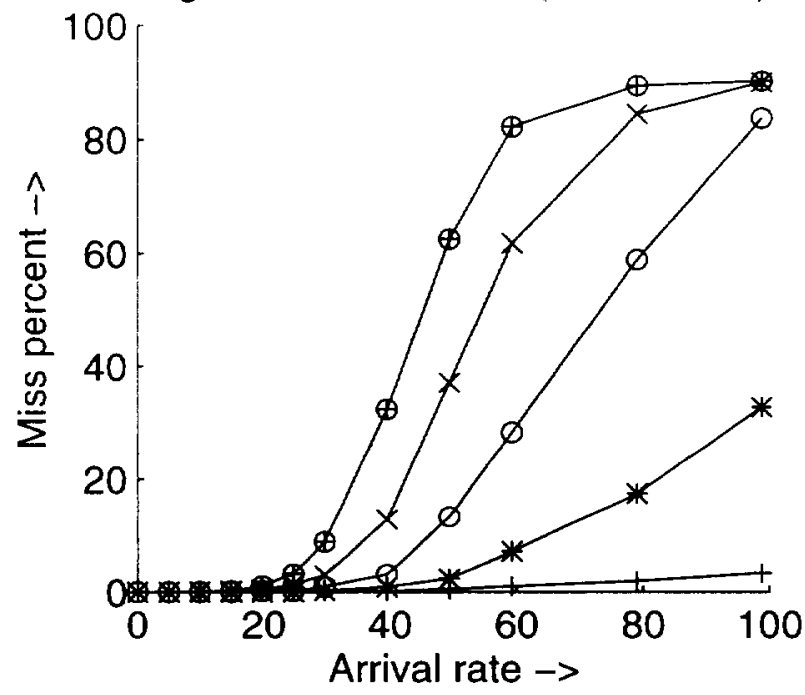

Figure 4d: Fairness (OPT-WAIT)

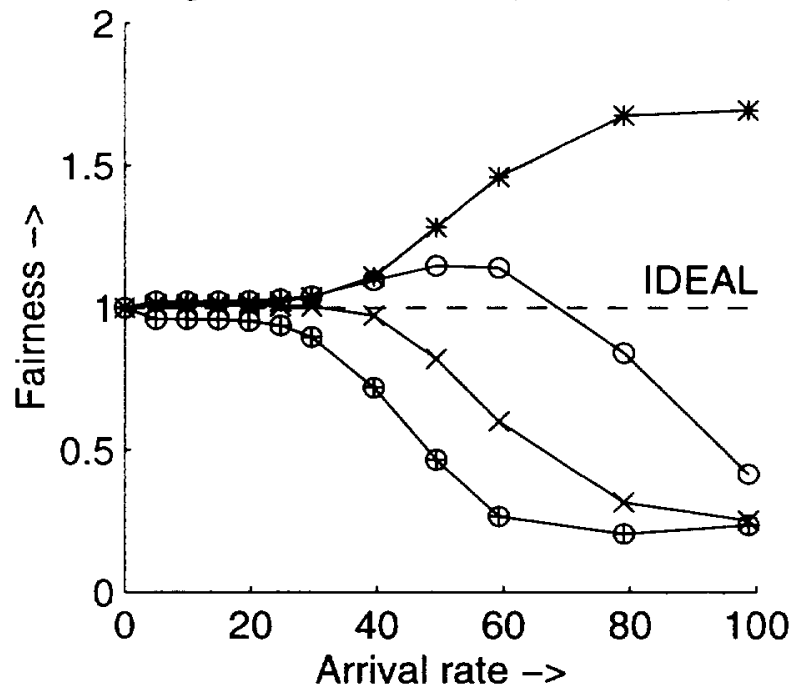


Figure 5a: Covert Security Cost

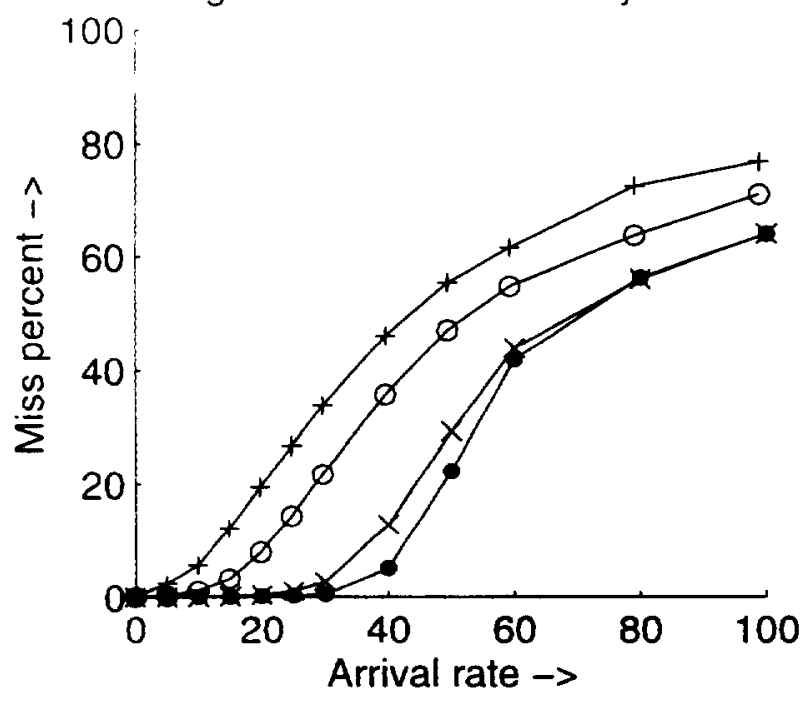

Figure 5c: Fairness

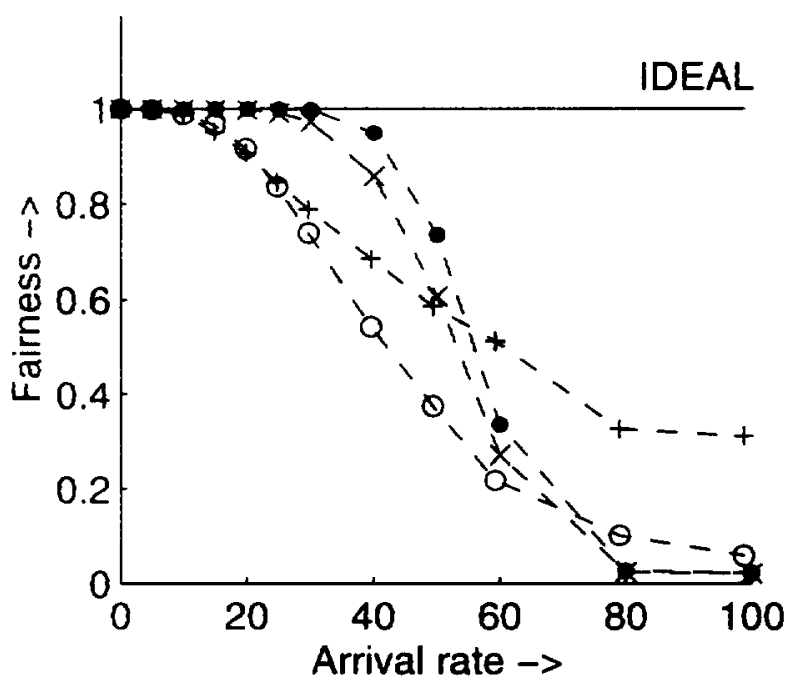

Hich wrthout morting to restarts unlike in WAIT-HP or

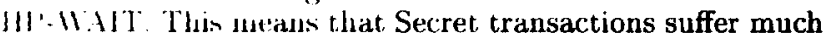
las in this environment (as confirmed in Figures $5 b$ and $5 c$ ). As she same time. using OPT-WAIT for handling intra- Jas contlicts helys to derive the inherent good real-time furfurmance associated with this protocol. At high loads, S2I'L-WAIT performs almost identically to OPT-WAIT berause. in this region, the primary reason for transactions musm then cheadlines is resource contention, rather than datic contcution therefore. the virtual commit feature of S2PL rarely provides the intended benefits.

In summary. this experiment shows that by carefully

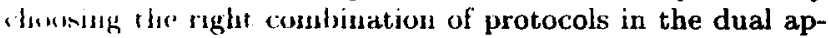
proach. we can design hybrid concurrency control algorithms that provide even better miss percent and fairness perforIniurr than (OPT-WAIT. This highlights the power and flexinlis. that is provided by the dual approach. In fact, it may lo pussible to develop hybrid algorithms that perform even better tilian S2PL-WAIT by appropriately choosing the constirtuent protocols
Figure 5b: Level miss percent
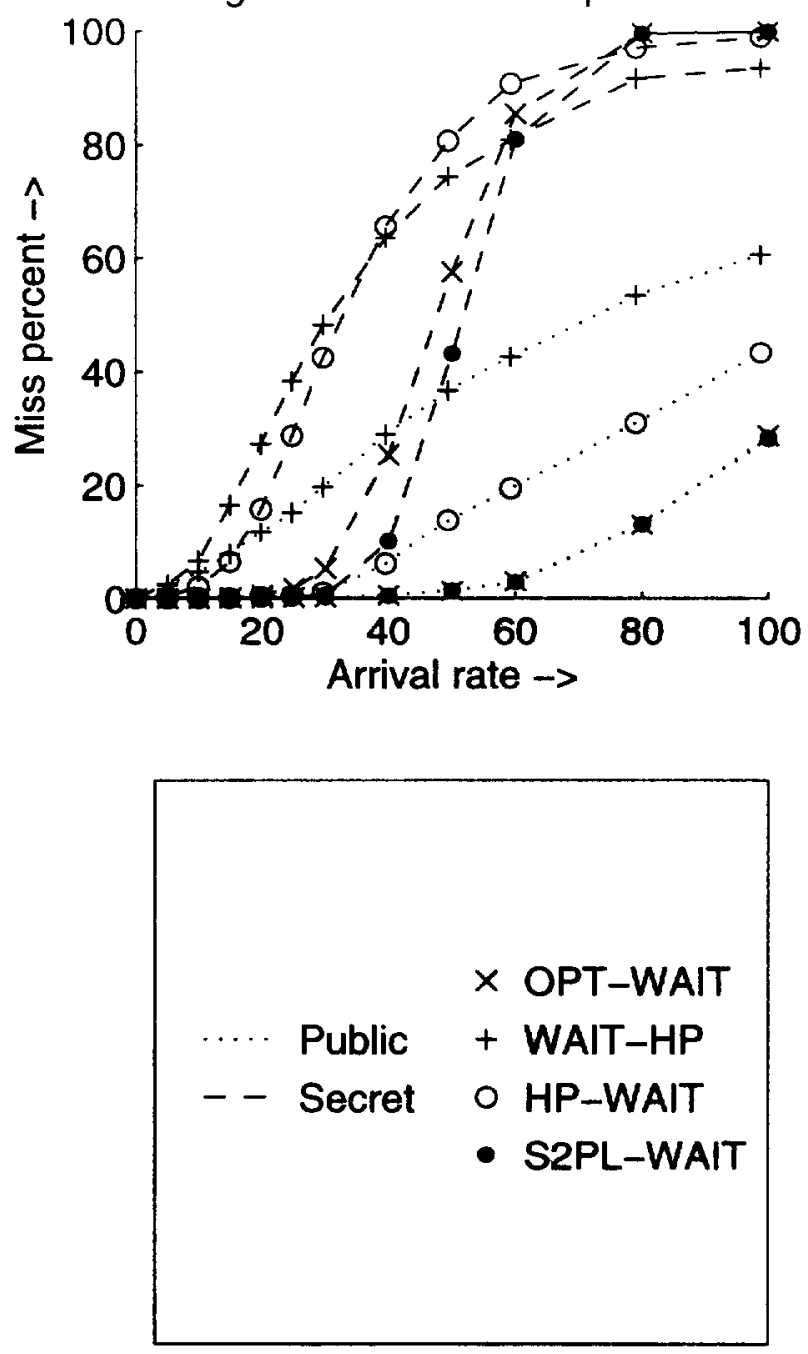

\section{Other Experiments}

We conducted several other experiments to explore various regions of the workload space. In particular, we evaluated the sensitivity of the results to the database size, number of security levels, deadline slack factor, etc. In many secure systems, the Bell-LaPadula model of "read below, write above" is further restricted to allow only "read below", that is, blind writes by low security transactions to high security data are disallowed. We conducted experiments to evaluate the performance behavior of the concurrency control protocols under this model also.

The complete details and results of the above experiments are available in [7]. Our general observation was that the relative performance behaviors of the protocols in these other experiments remained qualitatively similar to those seen in the experiments described here. That is, OPT-WAIT performed the best among the individual protocols, while S2PL-WAIT provided the best overall performance with respect to both the individual protocols and the dual combination protocols. 
hat this papex. Wre have quantitatively investigated the per-

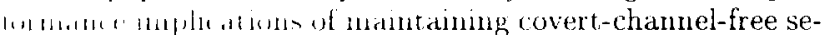
11114 !n a hru-risarthine real-time database system. Unhok promil: stmilts. which used a tradeoff approach bemron sinuty and timeliness, we have considered security if in "all-or-nothing" issue that is as a correctness criunil la comprumon, the number of missed deadlines is " performance issue. Therefore, our study investigates the prothlow of how to numinze the number of missed transacinss dratlunes untilout compromising security. To the best

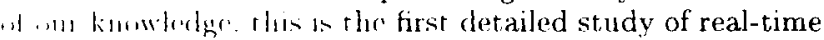
fuld abse serurity in the firm-deadline context

W1. tims dentified that, in order to satisfy the requiresucsu al uns-minterference. only those real-time concurrency initul profocols that are free from priority inversion can be Iis'd in il secure RTDBS. This requirement ruled out several previsuls mosesed real-time $\mathrm{CC}$ protocols, including algorithus stach as 2PL Wait Promote [3] and WAIT-50 [9].

Thin using a detailed simulation model of a firmwerdhu RIDBS. wo studied the relative performance of the wne versms of the 2PL-HP: OPT-SACRIFICE and OPTIIAIT real-time concurrency control algorithms; a non-realIsm, s'ure algorithm, S2PL, was also included in the evaluntion suite. The performance of these algorithms was also "sibiusterl for is baseline system where only direct unauthorized arcess. but not covert channels, is prevented.

(Our experinents showed that, under normal loads, the werall miss percent of the secure system is worse than that "if lhe liner svitem. whereas under heavy loads, it is the other wav around. Within the secure system, the perforusisur of high-seroriry transactions was significantly worse likan that of the low-security transactions. Among the se-

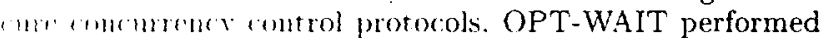
lent 14 mmimizing the miss percentages on both an overall basis and on a per-level basis. Moreover, it exhibited Ihe mitimum clegree of faimess. These results show that GTT. WAIT. which provided excellent performance in tra-

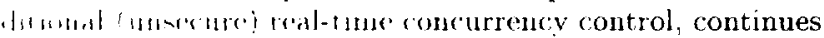
w profum well rern an the secure real-time domain.

Fillalli: we propesed a novel dual approach to secure comcurency control wherein different concurrency control dgenthus are used to resolve inter-level conflicts and intrakel coufticts. A global serialization order was ensured, in spirs of having multiple CC algorithms operating simultaucunsk. by using the system architecture proposed in [21]. Th" dual combluations of HP-WAIT and WAIT-HP were malluturalanl avaluated they both generally performed Wm, hall mar UPT-HAIT. However, the dual combinamu of $S 2 P L-W A I T$ performed better than OPT-WAIT, esmanils at lowes miss percent levels. This is because S2PL is a non-restiart-oriented algorithm unlike both OPT-WAIT and 2l'L-HP. and therefore ensures reduction of the harm "Wine to high-security transactions.

Awother advantage of the dual approach, not exploited here. in that the separation of security and timeliness con"'Tum matien it pussible to use even non-secure real-time ('(' algorithms (e.g.. Wait-Promote. WAIT-50) for resolving miri-lerel ronflicts! The dual approach therefore empowers the "Isi" "ven in the secure RTDBS domain, of the rich set of

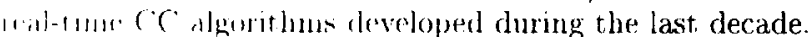

\section{Acknowledgements}

lhim wort was sipported in part by a research grant from the bern of sesemer and lechmologe. Govt. of India.

\section{References}

[1] R. Agrawal, M. Carey and M. Livny, "Concurrency control performance modeling: Alternatives and implications", ACM Trans. on Database Systems, 12(4), December 1987.

[2] D. Agrawal, A. El Abbadi and R. Jeffers, "Using Delayed Commitment in Locking Protocols for Real-Time Databases", Proc. of ACM SIGMOD Conf., June 1992.

[3] R. Abbott and H. Garcia-Molina, "Scheduling Real-time Transactions: A Performance Evaluation", ACM Trans. on Database Systems, September 1992.

[4] R. David, S. Son and R. Mukkamala, "Supporting Security Requirements in Multilevel Real-Time Databases", Proc. of IEEE Symp. on Security and Privacy, May 1995.

[5] K. Eswaran et al, "The Notions of Consistency and Predicate Locks in a Database Systems", Comm. of ACM, November 1976

[6] J. Goguen and J. Meseguer, "Security Policy and Security Models", Proc. of IEEE Symp. on Security and Privacy, 1982.

[7] B. George and J. Haritsa, "Secure Processing in Real-Time Database Systems", TR-97-02, DSL/SERC, Indian Institute of Science, 1997.

[8] I. Greenberg et al, "The Secure Alpha Study (Final Summary Report)", Tech. Report ELIN A012, SRI International, June 1993.

[9] J. Haritsa, M. Carey and M. Livny. "Data Access Scheduling in Firm Real-Time Database Systems", Real-Time Systems Journal, 4(3), 1992.

[10] J. Haritsa, M. Livny and M. Carey, "Earliest Deadline Scheduling for Real-Time Database Systems", Proc. of 12th IEEE Real-Time Systems Symp., December 1991.

[11] W. Lampson, "A Note on the Confinement Problem", Comm. of $A C M$, October 1973.

[12] L. LaPadula and D. Bell, "Secure computer systems: Unified Exposition and Multics Interpretation", The Mitre Corp., March 1976.

[13] R. Mukkamala and S. Son, "A Secure Concurrency Control Protocol for Real-Time Databases", Proc. of Annual IFIP WG 11.3 Conference of Database Security, August 1995.

[14] J. Robinson, "Design of concurrency control protocols for transaction processing systems", Ph.D. Thesis, Computer Sciences Dept., Carnegie Mellon University, 1982.

[15] S. Son and R. David, "Design and Analysis of a Secure TwoPhase Locking Protocol", Proc. of Intl. Computer Software and Applications Conf., November 1994.

[16] S. Son, R. David and B. Thuraisingham, "An Adaptive Policy for Improved Timeliness in Secure Database Systems", Proc. of Annual IFIP WG 11.3 Conference of Database Security, August 1995.

[17] S. Son, R. David, B. Thuraisingham, "Improving Timeliness in Real-Time Secure Database Systems", SIGMOD Record, Special Issue on Real-Time Database Systems, March 1996.

[18] L. Sha, R. Rajkumar and J. Lehoczky, "Priority inheritance protocols: an approach to real-time synchronization", Tech. Rep. CMU-CS-87-181, Depts. of CS, ECE and Statistics, Carnegie Mellon University, 1987.

[19] S. Son and B. Thuraisingham, "Towards a Multilevel Secure Database Management System for Real-Time Applications", Proc. of IEEE Workshop on Real-Time Applications, May 1993.

[20] B. Thuraisingham and H. Ko, "Concurrency Control in Trusted Database Management Systems: A Survey", SIGMOD Record, 22(4), December 1993.

[21] S. Thomas, S. Seshadri and J. Haritsa, "Integrating Standard Transactions in Real-Time Database Systems", Information Systems, 21(1), March 1996. 